\title{
„Das Herz ist der Schlüssel der Welt und des Lebens.“
}

\author{
Novalis (1772-1801), eigentlich Georg Philipp Friedrich Leopold Freiherr \\ von Hardenberg, deutscher Lyriker
}

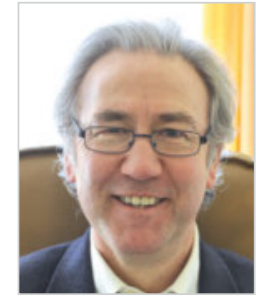

Volker Schmiedel
Korrespondenzadresse

Dr. med. Volker Schmiedel

Haldenstr. 1

$\mathrm{CH}-6340$ Baar

Bibliografie

DOI https://doi.org/10.1055/s-0043-109276

EHK 2017; 66: 197

(c) MVS Medizinverlage Stuttgart \& Co KG

ISSN 0014-0082
Würde man eine Umfrage nach dem wichtigsten Organ durchführen, so glaube ich, dass die meisten Menschen das Herz angeben würden. Es ist das einzige Organ, das wir auch im gesunden Zustand spüren können.

Wenn das Herz schwächelt, spüren wir das unmittelbar an Luftnot, Wassereinlagerungen und deutlich verminderter Leistungsfähigkeit. Während weniger als $1 \%$ der 45-55-jährigen daran leiden, sind es bei den 80 -Jährigen bereits fast $10 \%$. Da die Betroffenen ihre Medikamente lebenslang nehmen müssen, ist die Herzinsuffizienz ein durchaus lohnender Zukunftsmarkt. Da ist es auch nicht verwunderlich, dass immer neue Medikamente wie Angiotensin-Rezeptor/Neprilysin-Hemmer, Phosphodiesterase-III-Hemmer oder IKanal-Hemmer aus den Pipelines der Pharmaindustrie sprudeln. Mir fällt dabei auf, dass immer etwas „gehemmt“ werden muss. Müsste bei der Herzschwäche nicht eigentlich etwas „gefördert“ werden?

Was sagt denn die Leitlinie „Herzinsuffizienz“ der Deutschen Gesellschaft für Kardiologie von 2016 zu Naturheilverfahren bei dieser Krankheit? Wenn wir Digitalis einmal großzügig der Phytotherapie zurechnen wollen, dann hat diese alte vom britischen Arzt William Withering empirisch gefundene Behandlung in bestimmten Situationen auch heute noch ihren Stellenwert. Weißdorn existiert in der Kardiologie schlichtweg nicht.

Vonseiten der Orthomolekularen Therapie wären durchaus Vitamin D oder Q10 von Interesse - aber auch hier Fehlanzeige.

Immerhin wird eine Eisensubstitution empfohlen, wenn der Ferritinspiegel unter $100 \mu \mathrm{g} / \mathrm{I}$ sinkt. Omega-3-Fettsäuren können bei symptomatischen Herzinsuffizienzpatienten zur Senkung der kardiovaskulären Hospitalisationsrate und der kardiovaskulären Mortalität erwogen werden, was mit einem Evidenzgrad B versehen wurde. Aha, da haben also placebokontrollierte Doppelblindstudien ergeben, dass Herzinsuffiziente unter Omega-3-Fettsäuren signifikant weniger ins Krankenhaus müssen und eine geringere Sterberate aufweisen und der Kardiologe „kann es in Erwägung ziehen“. Realität ist, dass ich keinen einzigen Herzpatienten kenne, dessen Kardiologe jemals Omega-3-Fettsäuren empfohlen hätte.
Die Therapie mit Omega-3-Fettsäuren stellt damit die einzige leitliniengestützte Methode dar, die in diesem Heft in einer Übersicht mit vielen überzeugenden Studien gewürdigt wird. Omega3-Fettsäuren sollten danach nicht mehr erwogen werden, sondern es sollte ein Kunstfehler sein, sie nicht einzusetzen!

Dass ein 5000 Jahre altes komplexes Therapiesystem damals wie heute etwas zum Thema Herzschwäche zu sagen hat, belegt der Beitrag über ayurvedische Therapie.

Wir wissen heute, dass eine adäquate Bewegungstherapie sowohl Lebensdauer als auch -qualität zu verbessern vermag. Für einen Platz in der offiziellen Therapie reichen die Fakten den Leitlinienpäpsten aber leider noch nicht aus.

Die „guten alten“ Kneipp‘schen Verfahren Hydro- und Thermotherapie bieten Ansätze für eine ganzheitliche Herzinsuffizienzbehandlung, die man den Patienten nicht vorenthalten sollte.

Der von der Leitlinie unterschlagene Weißdorn wird in diesem Heft gebührend gewürdigt. Klinische Studien als auch praktische Erfahrung rechtfertigen den Einsatz dieser wirkungsvollen Phytotherapie.

Ein spannendes, medizinhistorisches Thema rundet mit der Entdeckung der Schüßler-Salze dieses „herzliche“ Heft ab.

Das Herz ist eines der ersten funktionierenden Organe in der Ontogenese überhaupt. Nach landläufiger Meinung endet das Leben, wenn das Herz aufhört zu schlagen. Insofern findet Tolstoi durchaus eine biologische Bestätigung:

\section{„Im Herzen eines Menschen ruht der Anfang und das Ende alle Dinge." \\ Leo Tolstoi (1828-1910), russischer Romanautor}

In diesem Sinne wünsche ich Ihnen, dass Sie auf das Herz Ihrer Patienten achten und verbleibe dieses Mal im wahrsten Sinne des Wortes mit herzlichen Grüßen

Dr. Volker Schmiedel 